\title{
Discrete mathematic analysis of the Dravnieks and Flavornet data bases
}

Terry Acree

\author{
From 1st International Workshop on Odor Spaces \\ Hannover, Germany. 4-7 September 2013
}

Odor Spaces to a flavor chemist are constructs represented by collections of chemicals with perceptual qualities. Their analytical procedures use chemistry to study the substances that generate Odor Spaces: foods, fragrances, and air quality. Psychologists view the same Odor Spaces as the sensations and perceptions invoked by chemicals and study these using the psychophysics of detection and the discrete mathematics of semantic memory. Neurobiologists, primarily using non-human models, study Odor Spaces by manipulating and measuring the physiology and genetics of the olfactory system. They have mostly focused on signal processing, however, "To understand the determinants of post-signal processing one needs to go beyond the bounds of olfactory research into the realm of cognitive theory." (W.S Cain). Those who study the synaptic organization of the brain and the psychology of perception are leading this effort.

This presentation will begin the Odor Spaces workshop with a discussion of the 1985 Dravnieks database compared to the 1997-2013 Flavornet database of odorants and their semantic descriptors. These and similar databases arise from a lifetime of experiences that give meaning to the Odor Spaces of the individuals, i.e. subjects, that generated them. Using elements of Graph theory both databases could be partitioned into a small subset of words that were associated with a large number of different chemicals indicating an odor class. A larger group of words each associated with a very small number of chemicals indicated a more specific relationship between chemical and sensation. That the class words were almost the same from the two different databases reveals a common process of classification used by two vastly different populations. This is likely a cogitative process; however, the very specific pairing of a majority of the chemicals to a few words may indicate a more peripheral mechanism.

Department of Food Science, Cornell University, 116 Stocking Hall, Ithaca, New York, USA 